\title{
COVID Vaccine Transport, Storage, and Distribution: Cold Chain Management to Ensure Efficacy
}

Michael Rusnack

AmericanPharma Technologies, Inc; (PharmaWatch)

\begin{abstract}
The Vaccines for Children Program (VFC) is a federally funded program in the United States, providing vaccines to children who lack health insurance or who otherwise cannot afford the vaccination cost. The VFC program was created in 1993 and is required to be a new entitlement of each State's Medicaid plan. The program was officially implemented in October 1994 and served eligible children in all United States (US). Other countries, the United Nations (UN), and the World Health Organization (WHO), have similar programs.

A critical aspect of these programs is the guidance surrounding the environmental monitoring of the materials. To best maintain the integrity of these products, specific storage parameters are required. It is necessary to store most vaccines at refrigeration or freezing temperatures. To best assure the efficacy of the vaccines, monitoring standards and equipment are specified. The technology and methodologies may be adequate for these programs' materials; these same methods are not for the COVID vaccine. ${ }^{[1]}$
\end{abstract}

When reviewing the guidance recommendations worldwide, one may observe commonalities in the program. Each guidance calls for the use of digital data loggers (DDL), sampling rates of 15 to 30 minutes, daily check-in (during business hours), and the use of a temperature buffer, each without specificity. ${ }^{[2]}$

The inadequacies of the VFC program monitoring methodologies fall far short when monitoring COVID vaccines. Herein considerations for the transport, storage, and distribution of the COVID vaccine cold chain will be discussed.

Keywords: COVID Vaccine Monitoring, Cold Chain Monitoring, Temperature Monitoring, Transportation, Temperature Buffer, Wireless Monitor, Internet of Things (IoT), Remote Monitoring

\section{OVERVIEW}

The Center for Disease Control and Prevention (CDC) gives guidance for vaccine storage and handling in the US. In conjunction with the National Institute of Standards and Technology (NIST), the CDC has developed the VFC monitoring program standards. These specifications have become the basis for much of the monitoring standards in pharmaceutical monitoring. The advancement of technology in sensing hardware being utilized is a break from the VFC standards. Given the higher value of the stored good (i.e., pharmaceuticals, medications, reagents, etc.), continuous monitoring is widely deployed. Advancements in wireless technology have enabled connectivity to cloud servers, allowing recorded data to be easily accessed.

Corresponding author: Michael Rusnack

Vice President of Science and Engineering

AmericanPharma Technologies, Inc

(PharmaWatch)

222 North 13th Street, Boise, ID 83702

Cell: 208.871 .9009
The use of a temperature buffer is specified in the VFC guidance; however, neither the volume nor geometry is defined. Similarly, the rate at which samples are recorded is quite broad and a throwback to the original data logger capacity. ${ }^{[3]}$ Despite the availability of continuous, real-time monitoring, daily check-in is a requirement. Here again, with automated systems that monitor $24 \times 7$, configurable alerting capable of voice, SMS (text), and email notification, users must record the temperature during work hours. The current "manual" system completely disregards any temperature excursions that may happen between daily check-in periods or on weekends and holidays.

Improper storage and handling results in as much as 35 percent of vaccines shipped worldwide wasted due to improper storage and transport. ${ }^{[4]}$ An audit by the Office of Inspector General noted these practices had been shown repeatedly to allow a substantial portion (30\% or more) of the vaccine inventory to be compromised without the practitioners being aware of this deficiency. ${ }^{[5]}$

While this is a problem for measles, it would be a catastrophe for a COVID-19 vaccine. Providentially, current technology provides an answer to this problem that can categorically assure the efficacy of the vaccine and notify the vaccine manager immediately of the potential of being compromised. 
The following describes the features of a "no compromise" state-of-the-art solution available with today's technology.

Much of this can be attributed to outdated equipment, methods, and procedures utilized. The VFC program and pharmaceutical monitoring represented the best available technology at the time of its implementation. The guidance has updated each year; however, it has failed to advance to the currently available technology and methodologies. Not all these spoiled vaccines and medications are discarded; they are distributed and utilized as viable. These results lead one to examine the consequence of such actions.

Logistics for pharmaceuticals have long experienced cold chain management-generally, keeping the materials at 2 to $8^{\circ} \mathrm{C}(35$ to $46^{\circ} \mathrm{F}$ ) during storage and transport. The advancement in recent years for cellular and genetic therapies, which involve live cells, has resulted in a growing need for deep-frozen storage: $-80^{\circ} \mathrm{C}$ $\left(-112^{\circ} \mathrm{F}\right)$ or even $-180^{\circ} \mathrm{C}\left(-292^{\circ} \mathrm{F}\right)$ requiring liquid nitrogen (LN2). It now appears that at least some of the certain COVID-19 vaccines will need to be stored at ultra-low temperatures. ${ }^{[7]}$

\section{MATERIALS AND METHODS}

Once they [cell and gene therapies] are on-site, human cells and the raw materials required for manufacturing must be carefully stored to maintain them. When assessing risk in these storage areas and implementing controls to prevent failure, manufacturers must consider that patient material is irreplaceable. ${ }^{[7]}$

\section{Real-Time Monitoring}

The protocols established for the VFC programs are to review and record the storage unit monitor's state twice daily during business hours, with no monitoring on weekends and holidays. Technological advancements in server architecture and wireless communication have enabled real-time monitoring and recordation. The result is a $24 \times 7$ data flow, which results in a continuously updated data presentation.

\section{Minimal Hardware Required}

With cloud computing, lower cost, scalable solutions are available to the end-user. Simply put, cloud computing is the delivery of computing services-including servers, storage, databases, networking, software, analytics, and intelligenceover the Internet ("the cloud") to offer faster innovation, flexible resources, and economies of scale. You typically pay only for cloud services you use, helping you lower your operating costs, run your infrastructure more efficiently, and scale as your business needs change. ${ }^{[8]}$

As a result of cloud computing, the integration of a monitoring solution is simplified. No longer is there a need for the expense of an on-site server. The facility and management costs are no longer a concern. The solution provider manages all of these services, eliminating the impact on the Information Technology (IT) department.

\section{Connectivity}

The path to the cloud servers is via the Internet. The two most common methods of connecting to the Internet include a direct connection (via LAN) and wireless. ${ }^{[9]} \mathrm{WiFi}$ is the most prevalent method for addressing data over the Internet. Both LAN and WiFi solutions require the services of the IT department.

In an existing facility where the WiFi network is established and operational, the IT department is burdened with:

- Different standards and protocols: Many types of wireless devices are used in the healthcare system. Multiple devices are integrated to monitor a single patient at times. The variance in these devices' standards and protocols make it difficult to integrate and interpret the data.

- Security and Privacy: The data between a doctor and a patient is bound by Doctor-patient confidentiality (HIPAA). The easy availability of and access to the data can compromise the security of both the patient and the doctor's personal health information. Additionally, weak security can allow the same access to the client network, exposing the healthcare system to ransomware.

- Data overload: Various devices are used with WiFi technology, all of which may not have the same communication protocols and standards. This can lead to a tremendous amount of data to be aggregated, all of which may not be effective in interpreting sensibly.

- System management: The IT department is now taxed with managing these connected devices on their already saturated network. System upgrades may now adversely affect these connected devices as they lag in the compliance and connectivity standards.

Though the burden is less with a LAN solution, these same issues overload the IT department as they work to accommodate the increased traffic. When outfitting a new facility, the complexity and expense of the solution are multiplied manifold. ${ }^{[10]}$

With IoT (Internet of Things) technology, coupled with the Internet's pervasiveness, a new sensing technology era, is ushered into use.

Definitions vary when describing loT:

- $\quad$ loT is about extending the Internet's power beyond computers and smartphones to a whole range of other things, processes, and environments. (IoT for All)

- IOT is the extension of internet connectivity into physical devices and everyday objects. Embedded with electronics, internet connectivity, and other hardware forms (such as sensors), these devices can 
communicate and interact with others over the Internet. They can be remotely monitored and controlled. (Wikipedia)

- $\quad$ IoT refers to a vast number of "things" connected to the Internet so they can share data with other things IoT applications, connected devices, industrial machines, and more. Internet-connected devices use built-in sensors to collect data and act on it. (SAS)

Cellular IoT provides wireless connectivity while being completely independent of the IT department; it should not be confused with WiFi enable loT devices as they open an entirely new and unique problem set.

\section{Sensors}

The data logger presently utilized for vaccine monitoring is not suited for monitoring temperatures below $-40^{\circ} \mathrm{C}\left(-40^{\circ} \mathrm{F}\right)$. These devices use a thermistor or digital temperature sensor. The accuracy of this family of devices at low temperatures decreases as they become very nonlinear-the data below the $-40^{\circ} \mathrm{C}\left(-40^{\circ} \mathrm{F}\right)$ is no longer viable.

Accurate measurements at these temperatures require the use of thermocouples or resistance temperature detectors (RTDs). There are pros and cons to each device. The thermocouple is a wire pair, with the sensing end either welded or twisted; the small form-factor allows for easy placement. Comparatively, the RTD sensing mechanism is typically encased in a stainlesssteel tube; this presents a challenge when placing the sensing probe.

The modern-day production of RTD and precision electronics results in a very accurate and stable measurement system. The thermocouple is susceptible to measurement error and requires frequent calibration. The calibration process is of the entire solution; this step requires either an on-site visit by a technician or complete removal and replacement of the monitor and probe. When the certification period is expired for the RTD, the probe is exchanged with minimal impact.

\section{Data Collection}

An artifact of the technology at the time, the CDC guidance, recommends a 30-minute sample rate. With no storage limitation, is the 30-minute sample rate optimal? To determine if the sample rate is optimized, it is necessary to oversample the typical storage unit's temperature change. The raw data from several refrigerator and freezer examples were analyzed using a Fourier Analysis algorithm. An average of a 10-minute sample interval was determined from this analysis. A five-minute sample interval is the recommended monitoring interval to ensure an accurate representation of the storage unit performance. Utilizing a 30-minute sample interval can and will result in lost data, i.e., possibly missing excursions, which would result in damaging the stored contents. ${ }^{[11]}$

\section{Data Presentation}

Bloomberg reported that United Parcel Service Inc. is building two giant freezer farms capable of super-cooling millions of vials of a COVID-19 vaccine, preparing for the day when it will need to deliver the medicine at high speed across the globe.

Under construction in Louisville, Kentucky, and the Netherlands, the facilities, near UPS air hubs, housing a total of 600 deep-freezers that can each hold 48,000 vials of vaccine at temperatures as low as -80 Celsius (-112 Fahrenheit). ${ }^{[12]}$

Newly purchased freezers areas susceptible to failure as one that has been in service for some time. Modern-day analytics built into today's monitoring solution can determine the consistency at which the goods are stored and monitor the freezer's performance. In these cases, the monitoring solution provides alert notifications for a temperature excursion and predictive analytics alerting the user of a potential failure.

Even in a facility with as few as ten refrigerators/freezers, it would be nearly impossible to accurately monitor each manually. Employing a monitoring solution in which the user can observe the monitoring conditions at-a-glance is essential.

The viewing hierarchy can be established to meet the customer's requirements. Below is an example of the Account / Location / Zone structure. An Administrator role may be allowed to view the entire entity, where a SuperUser with the responsibility of only a portion has less. A User may be designated with only a small number of zones in which they are responsible.

With a web-based display solution, the users may access the portal data from any device. These include PC, laptops, or mobile devices. The same hierarchy noted above for view access is configurable for alert notification. The alert notification process is explained in the following section of this manuscript. 


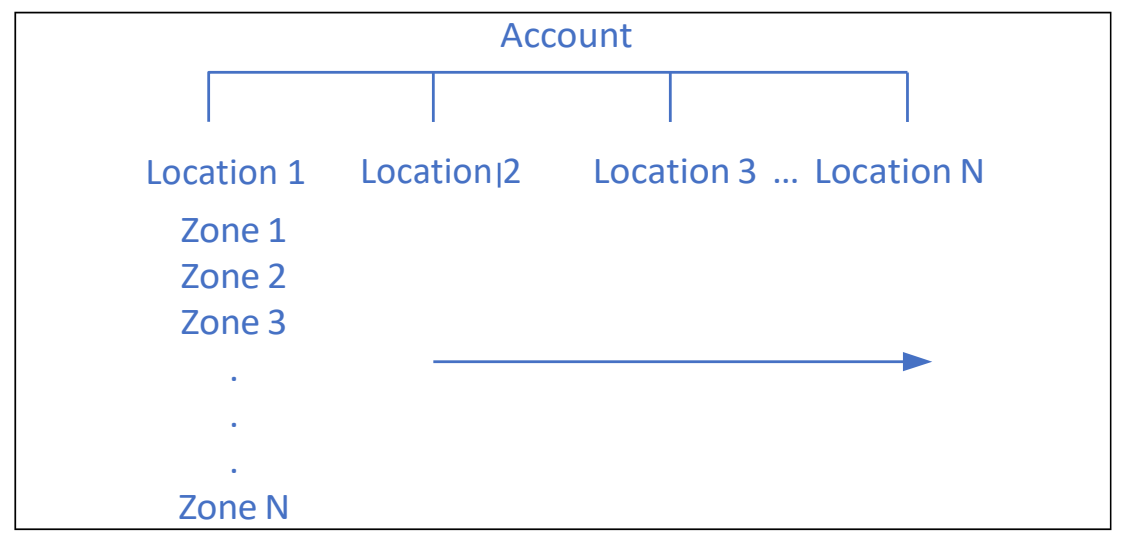

Figure 1 - Account Hierarchy Example

\section{(1) Midwest Depot Building 3}

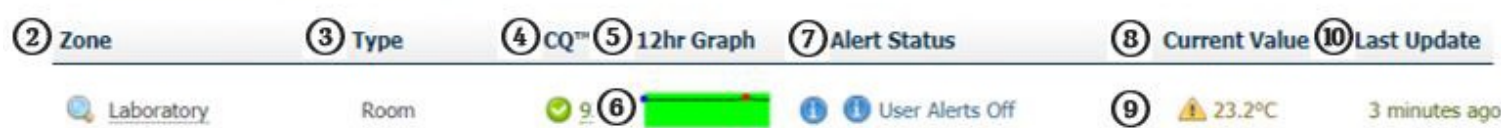

1. Top-level location - may be one of many on the dashboard.

2. Zone - a name by which the storage unit is referenced

3. Type - the type of equipment or facility being monitored

4. $\mathrm{CQ}^{\mathrm{TM}}$ - Compliance Quotient, a system performance report card

5. $12 \mathrm{hr}$ Graph - at-a-glance compliance to the specified control limits

6. The dot indicates low (blue) and high (red) values

7. Alert Status - Current alert state. Both System and User alerts are noted

a. Alerts off

b. Active

c. Reset

d. Non-reset

8. Current reading - the value of the most recent reading with the appropriate units

9. The colorized symbol indicates the device control chart state

10. Last Update - how long ago was the last reading

Figure 2 - Top Level Dashboard Explanation

Figure 3 is an example of an operational facility dashboard displaying several storage units being monitored. This graphic demonstrates the ability to display one to many locations to zones, in this case. A user with the necessary granted permissions can view an entire entity in a single pane in a single view. The dashboard is color-coded to enable the user to note any deviations outside of the control limits quickly. 
Midwest Depot Building 3

\begin{tabular}{|c|c|c|c|c|c|c|}
\hline Zone & Type & $\mathrm{CQ}^{\mathrm{mm}}$ & $12 \mathrm{hr}$ Graph & Alert Status & Current Value & Last Update \\
\hline Q Laboratory & Room & (7) 93 & $=$ & (1) (1) User Alerts off & A. $23.2^{\circ} \mathrm{C}$ & 3 minutes ago \\
\hline Q. Tank 07 & UltraCold & $\Delta 79$ & 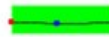 & No Open User Alerts & $-192.9^{\circ} \mathrm{C}$ & less than a minute ago \\
\hline Q. Tank 08 & UiltraCold & $\triangle 65$ & 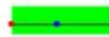 & No Open User Alerts & $-193.4^{\circ} \mathrm{C}$ & 1 minute ago \\
\hline Q Tank 09 & UltraCold & ( 93 & $\ldots$ & No Open User Alerts & $-197.0^{\circ} \mathrm{C}$ & 1 minute ago \\
\hline Q Tank 10 & UltraCold & (7) 93 & $\rightleftarrows$ & No Open User Alerts & $-197.2^{\circ} \mathrm{C}$ & 1 minute ago \\
\hline Q. Tank 11 & UltraCold & ( 93 & 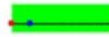 & No Open User Alerts & $-197.3^{\circ} \mathrm{C}$ & 1 minute ago \\
\hline Q. Tank 12 & UltraCold & ( 93 & 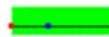 & No Open User Alerts & $-197.4^{\circ} \mathrm{C}$ & 1 minute ago \\
\hline Q. Tank 13 & UltraCold & 7 93 & & No Open User Alerts & $-197.1^{\circ} \mathrm{C}$ & 1 minute ago \\
\hline Q. Tank 14 & UltraCold & 93 & 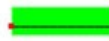 & No Open User Alerts & (- $-197.0^{\circ} \mathrm{C}$ & 1 minute ago \\
\hline Q. Tank 15 & UltraCold & (요 & & No Open User Alerts & $-197.2^{\circ} \mathrm{C}$ & 1 minute ago \\
\hline Q. Tank 16 & UltraCold & ( 93 & 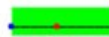 & No Open User Alerts & $-197.2^{\circ} \mathrm{C}$ & 1 minute ago \\
\hline Q TankA & UltraCold & ( 93 & . & No Open User Alerts & $-197.2^{\circ} \mathrm{C}$ & less than a minute ago \\
\hline
\end{tabular}

Figure 3 - Top Level Dashboard Expanded Example

\section{Alerting}

Data capture and reporting in the cloud allows the computing power to detect and process the alert notification. The client is notified of an out of control limit condition through alerting. Alert notifications are based on several states. The duration of the monitored condition is out of tolerance. Other possible requirements include:

- Low battery

- Loss of connectivity to the cloud

- Open / Short probe

- Missing probe

The VFC program's data logger notifies the user of an alert condition by a visual indication on the device or display. In some cases, an audible alarm is utilized. Both these methods require the user to be present and aware of being notified. In this age of low-cost cellular communication, an alert notification can include any of the following message and notification medium:

PharmaWatch STAFF USER ALERT

Med Clinic: Boise - Cole Road: Helmer

Temperature is at $3.3^{\circ} \mathrm{C}$, running critically above the alert limit of $3.1{ }^{\circ} \mathrm{C}$ detected Wed, August 12, 2020, 09:11

This email was generated Wed, August 12, 2020, 09:11.

- Email notification

- $\quad$ SMS (Text)

- Voice

The alert notifications can be sent to an unlimited number of registered users on the system. Each can process the alert in any of its four steps.
1) Acknowledgment - this step stops the alert notifications
2) Reset - once the alert condition is resolved, this step allows alerting
3) Documentation - allows the user to record the cause and resolution permanently
4) Close-completes the alert process

\section{Temperature Buffer Options}

It is common in the cold chain monitoring of pharmaceuticals, vaccines, tissue, and other temperature-sensitive materials to require a temperature buffer consisting of a physical container into which the temperature probe is inserted. When authoring this paper, there is no recommended or standard procedure for selecting this buffer. ${ }^{[13]}$ The physical buffer's specifics--size, shape, and material--are very rarely chosen consciously but established by default to whatever the company providing the temperature probe uses. A review of physical, thermal buffers currently offered by these companies shows little consistency in any of these parameters. Volumes can range from 10 to 300 $\mathrm{ml}$ glycol vials and machined and preformed aluminum blocks, plastic, or silicone. The Centers for Disease Control and Prevention recommends a $20 \mathrm{ml}$ Boston Bottle filled with an equal amount of water and glycol. The purpose of the water/glycol mixture is to prevent the freezing of the buffering solution. However, the stored goods monitored by this buffer were observed to include prefilled syringes as small as $0.25 \mathrm{ml}$ to bottles and vials many times that volume. ${ }^{[1][15]}$

The two purposes of the buffered temperature probe are somewhat in opposition to each other. A larger volume buffer prevents "false" alerting from transient changes caused by regular use; however, that same buffer may fail to respond quickly enough to freezing or warming events in time to prevent spoilage of the storage unit's contents. Both objectives can only be achieved when the buffer is thermally matched to the stored goods. 


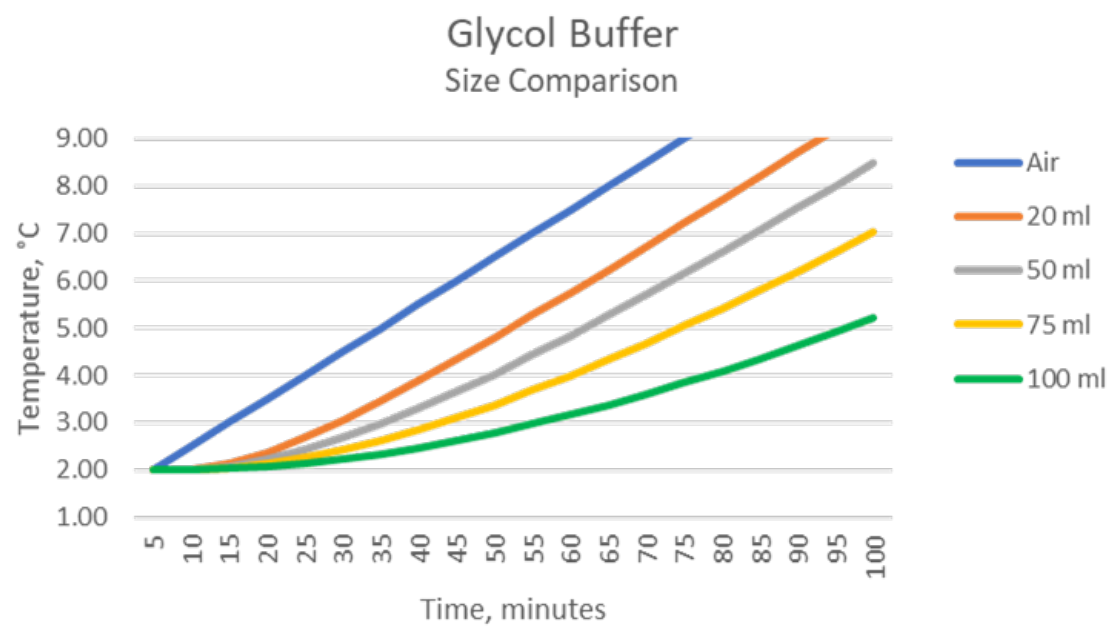

Figure 4 - Buffer Size Response Comparison (Warming) ${ }^{[14]}$

Figure 4 demonstrates the result of applying different volumes of glycol buffers to the same warming data. In this case, the warming of the refrigerator is shown (fault condition - door ajar). The difference between a $20 \mathrm{ml}$ and $50 \mathrm{ml}$ buffer reaching the 8.0-degree level is 15 minutes. For a $100 \mathrm{ml}$ buffer, this difference is 60 minutes. Accurate buffer sizing is critical when associating the measured temperature to that of the stored good. A similar observation is made when examining the same parameter for cooling conditions.
Figure $5 \mathrm{a}$ and Figure $5 \mathrm{~b}$ below demonstrates the effect of the selection of common buffer materials. In this presentation, the volume of the buffer remains constant while the content is changed. In each case, a $20 \mathrm{ml}$ volume of material is chosen. Aside from glycol, the results indicate that none of the selected buffer materials accurately represent the stored goods. To accurately represent the stored good, one must increase each of the other buffers' size to match the stored good characteristics. ${ }^{[14]}$

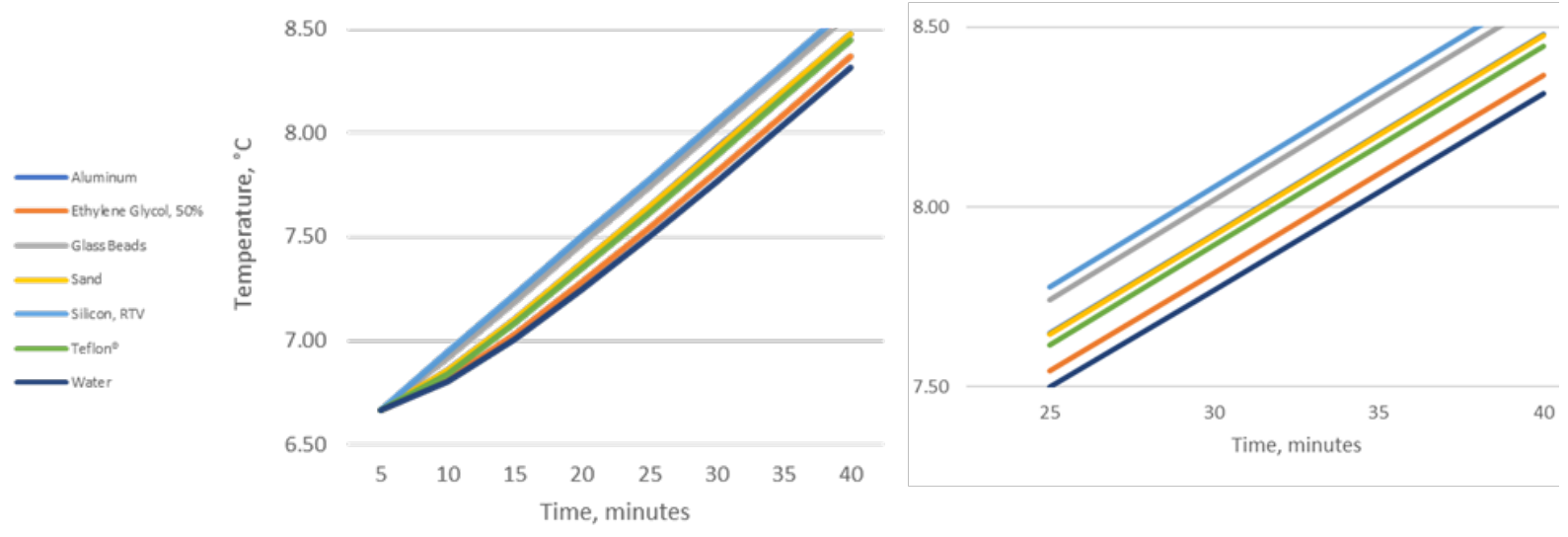

Figure 5 - Time to Temperature in Minutes for Various Buffer Material ${ }^{[14]}$

The geometry and material utilized for a physical buffer are critical to the accurate representation of the stored content. Additional consideration must be given when the storage temperatures are for deep-frozen storage: $-80^{\circ} \mathrm{C}\left(-112^{\circ} \mathrm{F}\right)$ or even $-180^{\circ} \mathrm{C}\left(-292^{\circ} \mathrm{F}\right)$. Paraffin wax is suggested as buffer material; this does not strictly represent the content being monitored. ${ }^{[15]}$
The selection of the thermal buffer used to monitor temperature-sensitive materials should not be left to the monitor system provider. It should be accomplished with great care and an understanding of the desired outcome. The following should be considered in the selection process: 
- What are the physical properties of the materials being stored?

- What is the range of package sizes being stored?

- What is the buffer material selected as the buffer?

Knowing these three factors, one can adequately size the buffer that will accurately represent the stored goods' temperature while providing valid and timely notification in a temperature excursion.

In those situations where the stored goods are packaged in various enclosures or vary from time to time, it is ultimately impossible to accomplish this. The option of Virtual Temperature Buffering (VTB) could be the best solution to these situations. VTB is an algorithm with the ability to model the stored good based on the air temperature.

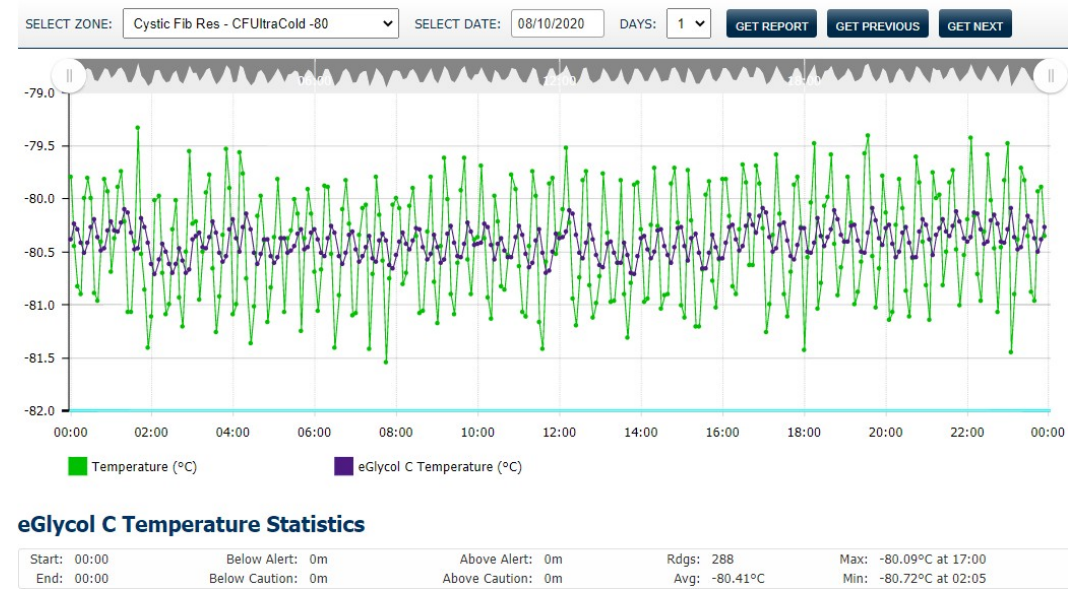

Figure 6 - Demonstration of Virtual Temperature Buffer (20 ml model)

Figure 6 above exhibits the result of the algorithmic buffer. The buffer being simulated in this case is a $20 \mathrm{ml}$ Boston Bottle geometry. With VTB, the air temperature remains unchanged and available for further processing while presenting the buffered value. Any geometry and contents can be modeled.

\section{Metrics, Objective and Subjective}

Data capture and reporting in the cloud allows performing data analytics on the gathered numbers. The simplest noted above, alerting when the measured value exceeds a predetermined threshold. Given the computing power available in the cloud servers, these complex computations may be accomplished without impacting the data gathering process.
Numerous reports and summaries are available based on the raw data collected. These reports may be necessary for regulatory compliance, maintenance records, or storage solution performance reports.

Figure 7 below shows a view of the day's measured data from a freezer with the summary statistics displayed below (see expanded window). The duration of any excursion above and below the control limits and average, minimum, and maxim values recorded are available to the user for the selected period. Also accessible is the Mean Kinetic Temperature (MKT) value for that period. The activation energy is programmable where needed. The MKT will aid the end-user in determining the potency of the product in the event of temperature excursions. 


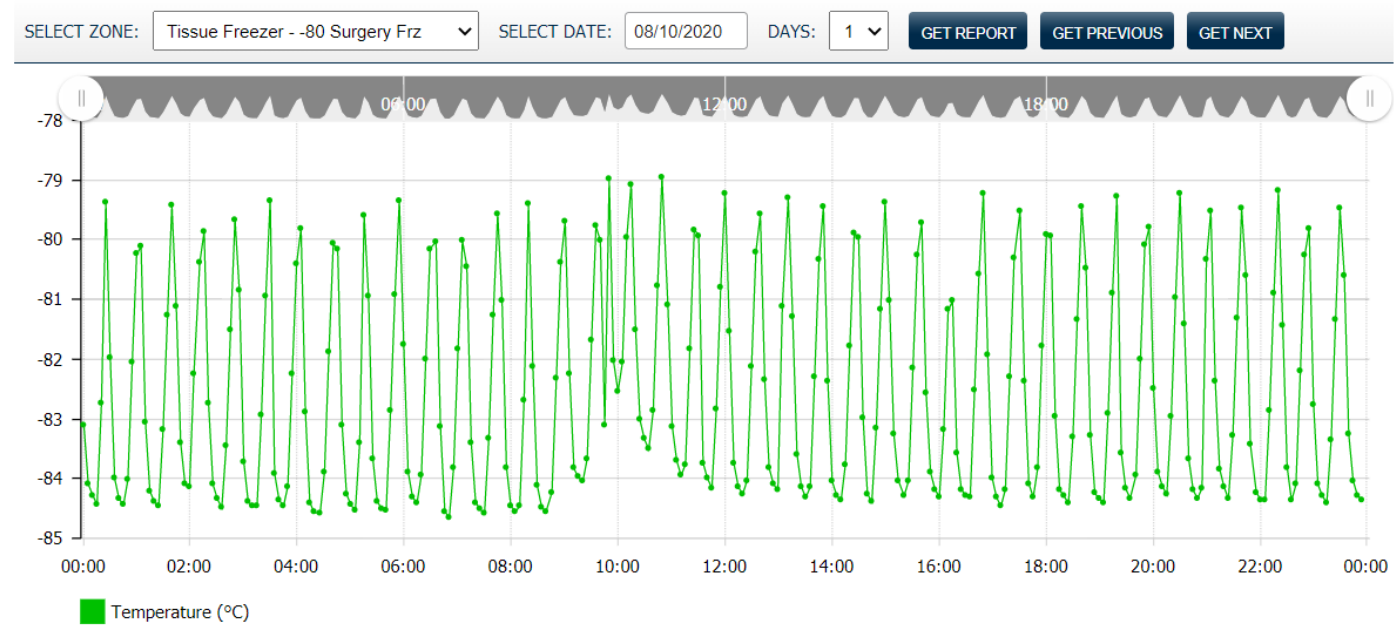

\section{Temperature Statistics}

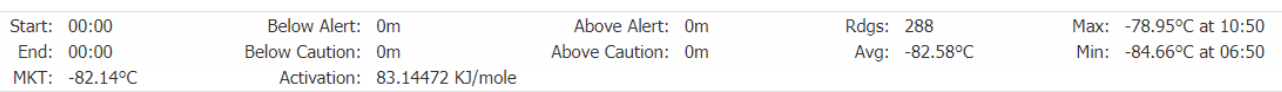

Figure 7 - Detail View Statistics

The data collected as part of the monitoring solution contains a wealth of ready harvesting information. Compliance with agency regulations can be reactive or proactive. A reactive response would follow the methodologies outlined for proper and accurate monitoring and only respond when alerted or otherwise notified. In contrast, proactive compliance would employ data analytics that provides the user with methods by which monitoring and management of the collected storage data may be accomplished. These methods would show strengths and weaknesses in monitoring and managing the overall compliance, thus allowing the user to address any potential liability and prevent loss due to non-compliance.

There are two primary contributors to potential noncompliance human and machine. In this case, the human refers to the user meeting the regulations' specifics in the mandated periodic data viewing and recordation, alert management, and documentation. The machine factors include the external environment, i.e., energy supply (AC Power), storage unit operation, and performance, to name a few. Each of these factors contributes to regulatory compliance in part and as an aggregate.

Any necessary and appropriate training can be implemented by recording and displaying the users to perform the required and mandated documentation. Albeit a small step, it results in adherence to regulatory guidance and better performance statistics. Similarly, machine performance can be measured and displayed. Predictive analytics may be utilized to monitor the storage unit. From this information, preventative maintenance may be cited to address an issue before It becomes serious. In the event of a power failure, the system can alert the user with sufficient time to prevent spoilage of the stored goods.
In quality improvement, managing data is an essential part of performance improvement. It involves collecting, tracking, analyzing, interpreting, and acting on an organization's data. Data management also includes ongoing measurement and monitoring. It is thus enabling an organization to identify and implement opportunities for improvements to the current process.

\section{DISCUSSION}

The cold chain distribution process Good Distribution Process (GDP) is the next step of the manufacturing program that all drugs and biological products are subject to and must adhere to health regulatory bodies. As such, the distribution process must be validated to ensure that there is no negative impact on the safety, efficacy, or quality of the vaccine in this case. ${ }^{[17]}$ The GDP environment's extension requires that all processes that might impact the safety, effectiveness, or quality of the vaccine must be validated, including the transportation, storage, and distribution of the vaccine. ${ }^{[17]}$

A failure of any point in the cold chain distribution process may result in the compromise vaccine. Without continuous and accurate monitoring throughout the process, a portion of the vaccine being distributed may become ineffective. When this occurs, some consequences may not be realized until after the vaccine is distributed and utilized. The observed consequence is a breakout of a cluster of vaccine-preventable illnesses.

UPS is building two facilities for the storage of the COVID vaccine. Each of the 600 freezers will store 48,000 vials of vaccine. ${ }^{[18]}$ The undetected failure of a single freezer can result in the distribution of ineffective vaccines to a population the size of a small city, leading to the vaccine not being effective. 
Confidence in the efficacy of the COVID vaccine is paramount. Present-day, trust in the routine childhood vaccinations is already shaken. As described above, an incident will only delay the need for a high percentage of vaccinating the public.

\section{CONCLUSION}

The cold chain includes the following "links":

- Transport from manufacturing facility to storage facility

- Warehousing of the vaccine in the storage facility

- Transport from the warehouse to localized distribution centers

- $\quad$ Storing of vaccines in preparation for distribution to clinics

- Transport from local distribution center to clinics

- Maintaining vaccines at clinics

- $\quad$ Transport and use at off-site (i.e., mobile clinics)

A break in any link of the above chain can result in the diminution of the stored vaccine. Responsibility for the supply chain monitoring will require continuity at all stages of the process. In the above scenario, the responsibility for the vaccine is broken into two entities. An example of this would-be UPS ${ }^{[18]}$ obtaining, storing, and distributing the vaccines to a large hospital provider that will, in turn, distribute the vaccine to community clinics. The community clinics may vaccinate their facility or offer an outreach program where the vaccines are transported and stored at a one-day clinic.

Cellular based IoT monitoring devices provide a unique opportunity to monitor the COVID vaccine cold chain. With no need to connect to a WiFi network, the system is plug and play. Recordation of each shipment is associated with the temperature data experienced by the contents of the container. The freezer farm's ongoing storage is monitored $24 \times 7$, assuring that any equipment failure, environmental anomaly, or human intervention is detected well before the vaccine is compromised. Real-time, end-to-end monitoring may be accomplished during transport, storage, and distribution. The raw temperature data can be analyzed, providing all analytics, quality measures, and reports.

Not unlike the above, the second phase of custody is monitored from a single dashboard. The hospital facility can maintain a close view of all storage facilities throughout its network. All of this ensures the safe and efficacious distribution of the COVID vaccine.

The solution described draws its parallels with the Vaccine for Children's program while advancing the use case and technology to the best available 2021. It is important to note that the VFC products' preponderance is maintained at 2 to $8^{\circ} \mathrm{C}$ (35 to $46^{\circ} \mathrm{F}$ ) during storage and transport. The leading candidates for the COVID vaccine will require deep-frozen storage: $-80^{\circ} \mathrm{C}\left(-112^{\circ} \mathrm{F}\right)$ or even $-180^{\circ} \mathrm{C}\left(-292^{\circ} \mathrm{F}\right)$, a completely different measurement system than that utilized for the VFC program.

The simplicity of the VFC program can be easily replicated, utilizing the methodologies described herein. It is necessary to maintain discipline in the monitoring during transport and storage. Of the many differentiators, cloud based reporting, real-time monitoring, and alert notification with data analytics, far surpass those features that are lacking in the VFC program.

\section{WORKS CITED}

[1] Your Current Monitoring May Not Be Good Enough For COVID19 Vaccines - Pharmawatch COVID, November 2020. https://www.pharmawatch.com/covid-19-vaccine/yourcurrent-monitoring-may-not-be-good-enough-for-covid-19vaccines/

[2] CDC. 2020. "Vaccine Storage and Handling Tookkit." January.

[3] NIST. n.d. "Reliable Vaccine Storage." Industry Impacts. https://www.nist.gov/industry-impacts/reliable-vaccinestorage.

[4] CDC.. 2020. "Vaccine Storage and Handling - Chapter 5." Epidemiology adn Prevention of Vaccine Preventable Diseases. July. https://www.cdc.gov/vaccines/pubs/pinkbook/vacstorage.html.

[5] Department of health and human services. "Vaccines for Children Program: Vulnerabilities in Vaccine Management." June 2012. https://oig.hhs.gov/oei/reports/oei-04-10- 00430.asp

[6]Chatterji, Saubjadra. 2020. "Govt plans to build cold storage, supply chain for COVID-19 Vaccines." Hindustan Times, July 27.

[7] Averall, Kasia. 2019. "Cell and Gene Therapies \& Thier GMP Requiremnts." ISPE. November / December. https://ispe.org/pharmaceutical-engineering/cell-genetherapies-their-gmp-requirements.

[8] Microsoft. 2020. What is Cloud Computing? https://azure.microsoft.com/en-us/overview/what-is-cloudcomputing/.

[9] Technopedia. 2019. "Internet of Things (IoT)." February 25. https://www.techopedia.com/definition/28247/internet-ofthings-

iot\#: :text=The\%20internet\%20of\%20things\%20\%28loT\%29\% 20is\%20a\%20computing,being\%20able\%20to\%20identify\%20t hemselves\%20to\%20other\%20devices.

[10] Julia Borgini, Spacebarpress Media. 2020. "Overcome 5 IoT device management challenges." TechTarget - IoT Agenda. June 8.

https://internetofthingsagenda.techtarget.com/tip/Overcom e-5-loT-device-management-challenges?track=NL1843\&ad=934659\&src=934659\&asrc=EM_NLN_129937239\& utm_medium=EM\&utm_source=NLN\&utm_campaign $=20200$ 623_Tackle\%20ICS\%20loT\%20security\%20challenges\%20wit h\%206. 
[11\} US Food Rusnack, M., 2018. Improving Vaccine Safety by Using an Algorithmic Model as a Replacement for a Physical Thermal Buffer. INNOVATIONS in pharmacy, 9(1), p.10. http://ttps://pubs.lib.umn.edu/index.php/innovations/article /download/962/1050

[12] Griffen, Riley and Koons, Cyntia. 2020. "Sanofi Partner Takes On Vaccine Hurdle: -112 Degree Storage." Bloomberg Law. May 20.

https://news.bloomberglaw.com/coronavirus/sanofipartner-takes-on-vaccine-hurdle-minus-112-degree-storage.

[13] Aldous, Jon. 2017. "How to select a Thermal Buffer from Glycol, Sand, Glass Beads,or Solid Material Blocks." Temperature Probe Buffer Types for Pharmaceutical \& Biotech Temperature Monitoring Applications. March 16. Accessed August 22, 2017. http://www.vaisala.com/en/lifescience/blog/Lists/Posts/Post .aspx?ID=149.
[14] Algorithm as a Replacement for a Physical Buffer." NCSL International . Portland, OR, ID: NCSL International

[15] Rusnack, Michael 2019. "Misuse and Misconceptions of Physical Temperature Buffering." NCSL International. Cleveland,OH: NCSL International Workshop and Symposium.

[16] Julie Steenhuysen, Kate Kelland. 2020. "Vaccine makers face biggest medical manufacturing challenge in history." Business News. June 2020. https://www.reuters.com/article/us-health-coronavirusvaccines-manufactu/vaccine-makers-face-biggest-medicalmanufacturing-challenge-in-history-idUSKBN23W1ND.

[17] Averall, Kasia. 2019. "Cell and Gene Therapies \& Thier GMP Requiremnts." ISPE. November / December. https://ispe.org/pharmaceutical-engineering/cell-genetherapies-their-gmp-requirements.

[18] Leffert, Catherine. 2020. "UPS Readies Freezer Farms to Ship Virus Vaccine - If We Get One." Bloomberg - Prognosis, August 3. https://www.bloomberg.com/news/articles/202008-11/putin-says-russia-has-registered-world-s-first-covid19-vaccine

\section{TABLE OF FIGURES}

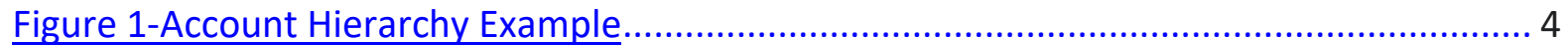

Figure 2 - Top Level Dashboard Explanation ............................................................ 4

Figure 3 - Top Level Dashboard Expanded Example .......................................................... 5

Figure 4 - Buffer Size Response Comparison (Warming) .................................................. 6

Figure 5 - Time to Temperature in Minutes for Various Buffer Material ...............................6 6

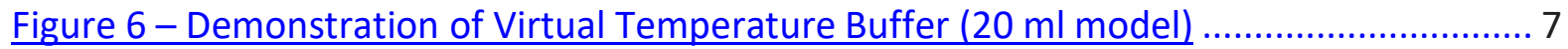

Figure 7 - Detail View Statistics ............................................................................................ 8 